\title{
Endoscopic Methods in the Diagnosis and Treatment of Pancreatic Cancer
}

\author{
Zoltán Berger*, Maria Ester Bufadel Godoy, Alex Navarro Reveco \\ University of Chile Clinical Hospital, Department of Medicine, Gastroenterology Section, Santiago, Chile. \\ Email: "berger.zoltan@gmail.com
}

Received July $15^{\text {th }}, 2013$; revised August $16^{\text {th }}, 2013$; accepted August $24^{\text {th }}, 2013$

Copyright @ 2013 Zoltán Berger et al. This is an open access article distributed under the Creative Commons Attribution License, which permits unrestricted use, distribution, and reproduction in any medium, provided the original work is properly cited.

\begin{abstract}
Endoscopic methods are widely used in the diagnosis and palliative treatment of pancreatic cancer. The most sensitive method in early diagnosis is endosonography (EUS) which can also provide histological diagnosis. Diagnostic ERCP became a rather rare procedure as a consequence of wide availability of Magnetic Resonance Cholangiopancreatography (MRCP) but ERCP assisted intraductal methods have gained importance (brush-cytology, intraductal ultrasound, optical coherence tomography) and finally, peroral pancreatoscopy has become technically feasible but available only in some specialized centers. Minimally invasive endoscopic methods play an important role in the palliative treatment of unresectable pancreatic cancer which represents the majority of cases. EUS-guided histological confirmation of adenocarcinoma is crucial in the election of chemotherapy. Celiac plexus blockade and endoscopic biliary and pancreatic stent placement contribute to pain reduction, drainage of obstructed bile duct and assure a better quality of life.
\end{abstract}

Keywords: Endoscopist; Early Diagnosis; Palliative Treatment

\section{Introduction}

Pancreatic cancer continues to be one of the most lethal malignancies: surgical resection is possible in only about $20 \%$ of detected cases and even in this group, the 5 year survival is low [1]. The diagnosis of cancer is established with conventional imaging procedures in the majority of these cases: CT-scan demonstrates the presence of the pathological mass and, at the same time, estimates the eventual vascular invasion, hepatic or peritoneal metastases, i.e. evident criteria of unresectability. If the tumor is considered resectable, surgery is the next step, without previous histological diagnosis except in those patients with clinical or radiological suspicion of another benign disease mimicking pancreatic cancer. Thus endoscopists face principally two extreme stages of pancreatic cancer: efforts in early diagnosis of small lesions and, on the other hand, palliative treatment of patients with advanced cancer.

\section{Endoscopy in the Early Diagnosis}

Thanks to systematic studies of surgical specimens, cancer precursor PanIN lesions have been identified. Unfor-

${ }^{*}$ Corresponding author. tunately, direct endoscopic access to visualize and resect these lesions - as in the case of adenomatous colon polyps-is not possible in everyday practice. A different emerging entity, pancreatic cystic lesions permit curative pancreatic surgery in initial phases of malignant transformation or even before, which consequently prevents cancer. However, early diagnosis of the most typical and most frequent adenocarcinoma of the pancreas continues to be unresolved. Five-year survival is far better in the subgroup of patients operated on with small malignant lesions, without metastasis. Early diagnosis is particularly important in genetically high-risk individuals. However, detection of small tumors-although not impossible-is extremely difficult.

There are some benign diseases of the pancreas which can produce tumor-like lesions or masses [2]. The differentiation of these diseases from pancreatic cancer is seldom easy. Our knowledge about the autoimmune pancreatitis has increased and differential diagnosis has become possible in the vast majority of these cases even without pancreatic biopsy [3,4]. Endoscopic methods, biopsy from the Vater papilla or even from gastric mucosa can be useful: $\operatorname{IgG}_{4}$ positive lymphoplasmocytic infiltration was found in every biopsy of Vater's papilla 
[5-7] and 12 of 13 cases of autoimmune pancreatitis in gastric mucosa [7]. These findings can be considered as histological proof of the $\operatorname{IgG}_{4}$ related benign disease with systemic involvement. Underdiagnosis of autoimmune pancreatitis can lead to unnecessary operations. On the other hand, "treatment" of pancreatic cancer with steroids could be the result of an opposite diagnostic error. Other benign disease, groove-pancreatitis [8] has no specific medical treatment and surgery is sometimes necessary.

\subsection{ERCP}

Pancreatography used to be the most sensitive method in detecting ductal changes [9] such as stenosis and consecutive dilatation of the pancreatic duct. Nevertheless, ERCP is an invasive method with potential complications, pancreatitis being the most frequent among them [10], which could make impossible to continue with the diagnostic work-up and delay the eventual surgery. MR cholangiography also provides excellent images of the pancreatic duct and its sensitivity seems comparable to that of ERCP [11]. However, cannulation of the pancreatic duct offers some advantages: we can obtain pancreatic juice or samples from the stenotic segments for brush cytology [12]. Pancreatic sphincterotomy and guide-wire placement also allow the introduction of special endoscopes ("babyscope") or accessories (spy-glass or intraductal ultrasound) in order to further investigate the lesions $[11,13,14]$.

ERCP does not add more information to a pancreatic focal lesion that was clearly demonstrated and characterized by other non-invasive images. In contrast, it can be useful to differentiate pancreatic cancer from benign inflammatory diseases which can produce ductal changes. Focal stenosis can be a part of inflammatory pancreatic diseases. However, in cancer patients upstream dilatation is almost always present with atrophy of surrounding parenchyma, but these changes are almost always lacking in autoimmune [3,4] or groove-pancreatitis [8]. Brush cytology can help to confirm the presence of cancer. Although specificity was high, the sensitivity of brush cytology has never been over $70 \%$ (only about $40 \%-50 \%$ in most publications) and its negative predictive value was about $30 \%$-reaching almost $50 \%$ in combination with intraductal biopsy [15]. In a recent publication, sensitivity of cytology was $65.8 \%$, specificity $100 \%$ and overall diagnostic accuracy $76.4 \%$ [12], with only 2 mild pancreatitis in 58 ERCPs. These values are quite similar to those obtained 10 years ago [16], in spite of progress in technology. Some additional advanced techniques can improve the results of cytology [17], but the negative predictive value remains low. Both brush cytology and intraductal forceps biopsy under fluoroscopic control are technically demanding and invasive methods, having a relatively high probability of sampling error. However, both are reasonably safe and represent a real possibility of early detection of some small malignant lesions. As expected, pancreatoscopy-guided tissue sampling has dramatically improved diagnostic accuracy and sensitivity [13] but this method is only exceptionally available.

\subsection{Endosonography (EUS)}

EUS provides an excellent high resolution image of the pancreas. Pancreatic cancer is seen as a hypoechoic inhomogeneous solid mass with irregular borders. The sensitivity of EUS in detection of pancreatic masses is somewhat superior to CT scan and MRI, particularly in the case of small lesions $[18,19]$. EUS alone is highly sensitive to demonstrate focal lesions and eventual vascular involvement. Using contrast-enhancement [20] with the Doppler method or digital image procession [21] can further improve its sensitivity. Diagnostic accuracy of EUS is over $80 \%$. It seems to be the method of choice to establish diagnosis and define unresectability, being cost-effective avoiding unnecessary and hopeless surgery. However, EUS is a more expensive, minimally invasive method with some complications; it is not widely available and is operator-dependent. In addition, its $60 \%$ $65 \%$ negative predictive value is relatively low, particularly in the presence of chronic pancreatitis and/or a previously implanted biliary stent [22]. As a consequence, CT scan continues to be the first method in the diagnostic work-up for pancreatic cancer, followed by EUS only in case of doubts in diagnosis or when biopsy is required. Linear EUS allows us to obtain biopsy specimens from virtually any pancreatic region, whatever its origin: not only from lesions related to the ductal system as is the case of brush cytology. On the other hand, although tumor seeding does exist [23] it is rather exceptional in spite of several passes as compared to US or CT-guided percutaneous biopsies. However, an acute inflammatory reaction provoked by EUS-FNA, can transform a small tumor into a nonresectable lesion [24]. EUS was also used to tattoo a small, otherwise undetectable lesion preoperatively and the small tumor was surgically resolved [25]. Marking the small tumor with silver pins provided the same result in another case reported [26].

Diagnostic sensitivity of FNA cytology improves with increasing tumor size, i.e. it is less useful in early diagnosis. The negative predictive value is also lower in the case of small lesions. This means that a negative cytology result does not exclude malignant nature of a small lesion and surgery is the next step even despite of negative cytology if the clinical suspicion is strong enough.

Like any other endoscopic technique, direct visualizetion of the pancreatic ductal system could be of great 
value. Thanks to pancreatoscopy, visualization is already a real possibility, although its availability is limited to rather few specialized centers. Apart from its cost and restricted availability, the small caliber of normal pancreatic duct represents a technical difficulty and limitation. Furthermore, tortuosity and strictures may prevent the scope from reaching the lesion in question. Intraductal US sondes [27] and intraductal optical coherence tomography [28] also offer greater precision to investigate ductal lesions and distinguish benign from malignant strictures, as compared to pancreatography. Utility of these methods is also limited to lesions originating in the main duct.

\section{Advanced Cancer}

Unfortunately, pancreatic cancer is advanced in the vast majority of cases at the time of diagnosis. Even relatively small $<2 \mathrm{~cm}$ lesions are not really early cancers; although resectable, lymph node metastasis, portal vein invasion and survival did not differ significantly as compared to major lesions [29]. The diagnosis is sometimes established during an ERCP performed for an obstructive jaundice of unknown cause. In such cases, if the obstructed bile duct is contrasted and probably contaminated, plastic stent placement is recommended. Self-expanding metal stents could probably interfere with posterior surgery [30]. In contrast, if the diagnosis of a resectable pancreatic cancer is established via other noninvasive methods, preoperative stent placement has no clinical utility, although it decreases the bilirubin-level [31].

Nowadays, the principal role of endoscopic methods is the palliative treatment of unresectable tumors.

1) Obstructive jaundice: This is one of the first clinical manifestations of tumors located in the head of the pancreas. Resolution of the obstruction and consecutive decrease in the jaundice reduces or completely controls pruritus. Technical success rate of endoscopic palliation is high. The placement of self-expanding metal endoprosthesis is the method of choice: they remain patent significantly longer than plastic stents. Obstruction and cholangitis are more frequent with plastic stents, needing emergency hospitalizations and repeated endoscopic interventions, thus deteriorating the quality of life. However, no difference was found in the survival in comparing polyethylene and metal stents [32]

2) Pain: The mechanism of pain in pancreatic cancer is complex and only partially understood. However, one of the components is probably the obstruction of the pancreatic duct. If pancreatography reveals a stricture with upstream dilatation it seems reasonable to place a plastic stent through the stricture and drain the obstructed pancreatic segment. Insufficient literature data prevent mak- ing definitive conclusions, but several results support the usefulness of pancreatic stents in the treatment of the pain [33]. There are also some initial results with radioactive stents placed either in the common bile duct or in the pancreatic duct which could stabilize the disease and delay progression [34]. Metal stents covered by a paclitaxel-incorporated polyurethane membrane gave similar results in 9 patients with bile duct strictures caused by unresectable pancreatic cancer [35].

3) Gastric outlet obstruction, duodenal stenosis: Simultaneous or consecutive obstruction of the bile duct and duodenum has been the cause of relatively complex surgical interventions in advanced pancreatic cancer (gastro-jejunal anastomosis and hepatico-jejunostomy). In addition, these are frequently a high surgical risk patients or even unfit for operations. Self-expanding metal stent placement by endoscopy [36] reestablishes almost normal caliber duodenal lumen, avoids vomiting and allows although somewhat limited but oral feeding. It also makes posterior endoscopic access to the papilla possible for the treatment of obstructive jaundice.

\subsection{Endosonography (EUS)}

1) EUS is the most sensitive method in the diagnosis of focal pancreatic lesions. However, in the case of advanced cancer the pancreatic mass has already been detected and declared unresectable by other methods. There are two questions to answer: a) Is the mass a ductal adenocarcinoma or of different histological nature? b) Is the patient fit for chemotherapy or another minimally invasive palliative treatment? If the patient's general condition does not allow even minimally invasive interventions and chemotherapy is considered unsuitable, the only possibility is conservative symptomatic treatment. Histological diagnosis is useless in these cases. On the other hand, except in the terminal stage, histological confirmation of the lesion is recommended to exclude benign diseases mimicking pancreatic cancer or malignant lesions different from adenocarcinoma, which may require different treatment and have better prognosis. The best way to obtain histological samples at present is the EUS-guided fine-needle biopsy [37-39], with high diagnostic accuracy and low risk of complications, including tumor seeding. The only argument against EUS is the cost and lack of wide availability in several countries.

2) EUS guided interventions:

a) Celiac plexus blockade (CPB) and neurolysis (CNL) are safely performed when guided by EUS. Results in pain control are similar to the radiologically-guided percutaneous or surgical interventions: CPB and/or CNL permit a reduction in the dose of analgesic drugs and result in better pain control. Published complications have been mild and transient [40]. 
b) EUS-guided drainage can facilitate pancreatic or biliary stent placement via "rendez-vous" techniques. Bilio-digestive drainage, choledocho-duodenostomy with stent placement can also be performed in cases of duodenal stenosis.

c) There are also some rather experimental interventions guided by EUS. Jin et al. [41] implanted iodine-125 seeds inside of unresectable pancreatic tumors of 22 patients. Combined with traditional chemotherapy, partial remission was achieved in 3 and stabilized the disease in another 10 patients, while pain diminished in 18 of the 22 patients with a statistically significant decrease in pain intensity as measured by visual analogue scale. Half of the patients presented fever which was treated with antibiotics, but no other serious complication was observed. EUS was used to guide intratumoral injection of a virus: ONYX-015, which selectively replicates in and destroys tumor cells, that are deficient in p53 function but not in cells with functional p53 [42,43]. This treatment was also associated with gemcitabine and the cancer did not progress in 8 of 21 patiens who participated in the study.

\section{Conclusion}

Algorithm of diagnosis and treatment of pancreatic cancer is depicted in Figure 1. Early diagnosis has somewhat improved but has not been resolved. The best, most sensitive and more and more widely available method for early detection is the EUS, with or without FNA, but it is not good enough. Its negative predictive value has improved in recent years, but surgery must be performed in spite of negative EUS and cytology results if the clinical suspicion is strong. More direct methods to visualize ductal lesions are in progress. On the other hand, endoscopic methods play an increasingly important role in

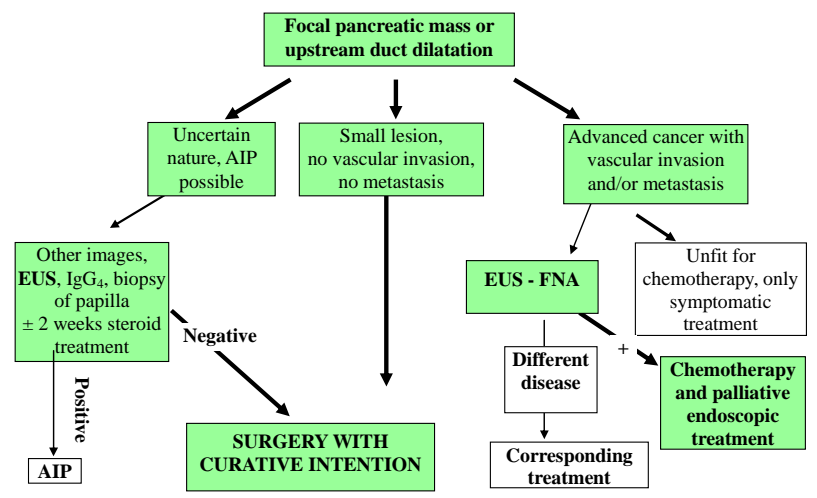

Figure 1. Endoscopic methods in the algorithm of diagnosis and treatment of pancreatic cancer. Pancreatic lesion initially detected with generally non-invasive imaging (CT, RM), rarely with ERCP. EUS is the best method in the differential diagnosis, with or without FNA. Other endoscopic methods have their dominant role in the palliative treatment of advanced lesions. the palliative treatment of pancreatic cancer patients. Self-expanding metal duodenal and biliary stents represent a good alternative treatment to replace relatively complex surgery in patients with less than 1 year life expectancy.

\section{REFERENCES}

[1] P. Michl, S. Pauls and T. M. Gress, "Evidence-Based Diagnosis and Staging of Pancreatic Cancer," Best Practice \& Research Clinical Gastroenterology, Vol. 20, No. 2, 2006, pp. 227-251. http://dx.doi.org/10.1016/j.bpg.2005.10.005

[2] G. Zamboni, P. Capelli, A. Scarpa, G. Bogina, A. Pesci, E. Brunello and G. Klöppel, "Nonneoplastic Mimickers of Pancreatic Neoplasms," Archives of Pathology \& Laboratory Medicine, Vol. 133, No. 3, 2009, pp. 439-453

[3] S. T. Chari, N. Takahashi, M. J. Levy, T. C. Smyrk, J. E. Clain, R. K. Pearson, B. T. Petersen, M. A. Topazian and S. S. Vege, "A Diagnostic Strategy to Distinguish Autoimmune Pancreatitis From Pancreatic Cancer," Clinical Gastroenterology and Hepatology, Vol. 7, No. 10, 2009, pp. 1097-1103. http://dx.doi.org/10.1016/j.cgh.2009.04.020

[4] T. Kamisawa M. Imai, P.-Y. Chen, Y. Tu, N. Egawa. K. Tsuruta, A. Okamoto and N. Suzuki Kamata, "Strategy for Differentiating Autoimmune Pancreatitis from Pancreatic Cancer," Pancreas, Vol. 37, No. 3, 2008, pp. e62e67

[5] P. Sahin, J. Pozsár, K. Simon, Gy. Illyés, F. László and L. Topa, "Autoimmune Pancreatitis Associated with Immune-Mediated Inflammation of the Papilla of Vater. Report on Two Cases,” Pancreas, Vol. 29, No. 2, 2004, pp. 162-166. http://dx.doi.org/10.1097/00006676-200408000-00012

[6] T. Kamisawa, Y. Tu, N. Egawa, K. Tsuruta and A. Okamoto, "A New Diagnostic Endoscopic Tool for Autoimmune Pancreatitis,” Gastrointestinal Endoscopy, Vol. 68, No. 2, 2008, pp. 358-361. http://dx.doi.org/10.1016/j.gie.2008.02.018

[7] T. Kamisawa, N. Egawa, H. Nakajima, K. Tsuruta, A. Okamoto, Y. Hayashi and N. Funata, "Gastrointestinal Findings in Patients with Autoimmune Pancreatitis," Endoscopy, Vol. 37, No. 11, 2005, pp. 1127-1130. http://dx.doi.org/10.1055/s-2005-870369

[8] A. H. de Tejada, J. Chennat, F. Miller, T.Stricker, J. Matthews and I. Waxman, "Endoscopic and EUS Features of Groove Pancreatitis Masquerading as a Pancreatic Neoplasm,” Gastrointestinal Endoscopy, Vol. 68, No. 4, 2008, pp. 796-798. http://dx.doi.org/10.1016/j.gie.2008.02.015

[9] R. J. Shah and S. P. Martin, "Endoscopic Retrograde Cholangiopancreatography in the Diagnosis and Management of Pancreatic Diseases,” Current Gastroenterology Reports, Vol. 2. No. 2, 2000, pp.133-145. http://dx.doi.org/10.1007/s11894-000-0097-6

[10] P. B. Cotton, D. A. Garrow, J. Gallagher and J. Romagnuolo, "Risk Factors for Complications after ERCP: A 
Multivariate Analysis of 11,497 Procedures over 12 Years,” Gastrointestinal Endoscopy, Vol. 70, No. 1, 2009, pp. 80-88. http://dx.doi.org/10.1016/j.gie.2008.10.039

[11] D. Domagk, J. Wessling, P. Reimer, L. Hertel, C. Poremba, N. Senninger, A. Heinecke, W. Domschke and J. Menzel, "Endoscopic Retrograde Cholangiopancreatography, Intraductal Ultrasonography, and Magnetic Resonance Cholangiopancreatography in Bile Duct Strictures: A Prospective Comparison of Imaging Diagnostics with Histopathological Correlation,” The American Journal of Gastroenterology, Vol. 99, No. 9, 2004, pp. 16841689.

http://dx.doi.org/10.1111/j.1572-0241.2004.30347.x

[12] N. Uchida, H. Kamada, K. Tsutsui, M. Ono, Y. Aritomo, T. Masaki, Y. Kushida, R. Haba, T. Nakatsu and S. Kuriyama, "Utility of Pancreatic Duct Brushing for Diagnosis of Pancreatic Carcinoma,” Journal of Gastroenterology, Vol. 42, No. 8, 2007, pp.657-662. http://dx.doi.org/10.1007/s00535-007-2071-7

[13] S. Iqbal and P. D. Stevens, "Cholangiopancreatoscopy for Targeted Biopsies of the Bile and Pancreatic Ducts," Gastrointestinal Endoscopy Clinics of North America, Vol. 19, No. 4, 2009, pp. 567-577.

http://dx.doi.org/10.1016/j.giec.2009.06.005

[14] K. V. Chathadi and Y. K. Chen, "New Kid on the Block: Development of a Partially Disposable System for Cholangioscopy," Gastrointestinal Endoscopy Clinics of North America, Vol. 19, No. 4, 2009, pp. 545-555. http://dx.doi.org/10.1016/j.giec.2009.06.001

[15] G. Elek, T. Gyökeres, E. Schäfer, M. Burai, F. Pintér and Á. Pap, "Early Diagnosis of Pancreatobiliary Duct Malignancies by Brush Cytology and Biopsy,” Pathology Oncology Research, Vol. 11, No. 3, 2005, pp. 145-155. http://dx.doi.org/10.1007/BF02893391

[16] J. Vandervoort, R. M. Soetikno, H. Montes, D. R. Lichtenstein, J. Van Dam, F. W. Ruymann, E. S. Cibas and D. L. Carr-Locke, “Accuracy and Complication Rate of Brush Cytology from Bile Duct versus Pancreatic Duct," Gastrointestinal Endoscopy, Vol. 49.No. 3 1999, pp. 322-327. http://dx.doi.org/10.1016/S0016-5107(99)70008-8

[17] L. E. Moreno Luna, B. Kipp, K. C. Halling, T. J. Sebo, W. K. Kremers, L. R. Roberts, E. G. Barr Fritcher, M. J. Levy and G. J.Gores, “Advanced Cytologic Techniques for the Detection of Malignant Pancreatobiliary Strictures,” Gastroenterology, Vol. 131, No. 4, 2006, pp. 1064-1072.

http://dx.doi.org/10.1053/j.gastro.2006.08.021

[18] S. Pappas, M. P. Federle, A. E. Lokshin and H. J. Zeh, "Early Detection and Staging of Adenocarcinoma of the Pancreas,” Gastrointestinal Endoscopy Clinics of North America, Vol. 36, No. 2, 2007, pp. 413-429. http://dx.doi.org/10.1016/j.gtc.2007.03.013

[19] R. H. Hruban, A. Maitra, S. E. Kern and M. Goggins, "Precursors to Pancreatic Cancer," Gastroenterology Clinics of North America, Vol. 36. No. 4, 2007, pp. 831849.

[20] C. F. Dietrich, A. Ignee, B. Braden, A. P. Barreiros, M. Ott and M. Hocke, "Improved Differentiation of Pancre- atic Tumors Using Contrast-Enhanced Endoscopic Ultrasound," Clinical Gastroenterology and Hepatology, Vol. 6, No. 5, 2008, pp. 590-597.

[21] A. Das, C. C. Nguyen, F. Li and B. Li, "Digital Image Analysis of EUS Images Accurately Differentiates Pancreatic Cancer from Chronic Pancreatitis and Normal Tissue,” Gastrointestinal Endoscopy, Vol. 67, No. 6, 2008, pp. 861-867. http://dx.doi.org/10.1016/j.gie.2007.08.036

[22] N. B. Krishna, M. Mehra, A. V. Reddy and B. Agarwal, "EUS/EUS-FNA for Suspected Pancreatic Cancer: Influence of Chronic Pancreatitis and Clinical Presentation with or without Obstructive Jaundice on Performance Characteristics," Gastrointestinal Endoscopy, Vol. 70, No. 1, 2009, pp. 70-79. http://dx.doi.org/10.1016/j.gie.2008.10.030

[23] S. C. Paquin, G. Gariépy, L. Lepanto, R. Bourdages, G. Raymond and A. V. Sahai, "A First Report of Tumor Seeding Because of EUS-guided FNA of a Pancreatic Adenocarcinoma,” Gastrointestinal Endoscopy, Vol. 61, No. 4, 2005, pp. 610-611.

[24] S. Dahl and M. B. Mortensen, "Endoscopic Ultrasound-Guided Fine-Needle Aspiration Can Lead to Nonresectability of Pancreatic Cancer Due to Severe Biopsy-Induced Inflammation,” Endoscopy, Vol. 40, Suppl. 2, 2008, p. E96.

[25] J. J. Farrell, A. Sherrod and D. Parekh, "EUS-Guided Fine-Needle Tattooing for Preoperative Localization of Early Pancreatic Adenocarcinoma," Gastrointestinal Endoscopy, Vol. 69, No. 1, 2009, pp. 176-177. http://dx.doi.org/10.1016/j.gie.2008.03.1069

[26] M. H. Larsen, C. W. Fristrup and M. B. Mortensen, “Endoscopic Ultrasound-Guided Fine-Needle Marking of a Small Pancreatic Tumor,” Endoscopy, Vol. 41, Suppl. 2, 2009, pp. E175-176. http://dx.doi.org/10.1055/s-0029-1214699

[27] R. Kundu and D. Pleskow, "Clinical Application of Intraductal Ultrasound during Endoscopic Retrograde Cholangiopancreatography," Gastrointestinal Endoscopy Clinics of North America, Vol. 19, No. 4, 2009, pp. 615-628. http://dx.doi.org/10.1016/j.giec.2009.06.004

[28] P. A. Testoni, A. Mariani, B. Mangiavillano, P. G. Arcidiacono and S. E. Di Pietro, "Intraductal Optical Coherence Tomography for Investigating Main Pancreatic Duct Strictures," The American Journal of Gastroenterology, Vol. 102, No. 2, 2007, pp. 269-274.

[29] K. C. Chiang, C. N. Yeh, W. C. Lee, Y. Y. Jan and T. L. Hwang, "Prognostic Analysis of Patients with Pancreatic Head Adenocarcinoma Less Than $2 \mathrm{~cm}$ Undergoing Resection,” World Journal of Gastroenterology, Vol. 15, No. 34 ,2009, pp. 4305-4310.

http://dx.doi.org/10.3748/wjg.15.4305

[30] Pancreatic Section of the British Society of Gastroenterology, Pancreatic Society of Great Britain and Ireland, Association of Upper Gastrointestinal Surgeons of Great Britain and Ireland, Royal College of Pathologists, Special Interest Group for Gastro-I: “Guidelines for the Management of Patients with Pancreatic Cancer Periampullary and Ampullary Carcinomas," Gut, Vol. 54, Suppl. 5, 
2005, pp. 1-16. http://dx.doi.org/10.1136/gut.2004.057059

[31] J. J. Mezhir, M. F. Brennan, R. E. Baser, M. I. D’ Angelica, Y. Fong, R. P. Dematteo, W. R. Jarnagin and P. J. Allen, "A Matched Case-Control Study of Preoperative Biliary Drainage in Patients with Pancreatic Adenocarcinoma: Routine Drainage Is Not Justified," Journal of Gastrointestinal Surgery, Vol. 13, No. 12, 2009, pp. 2163-2169. http://dx.doi.org/10.1007/s11605-009-1046-9

[32] A. Weber, T. Mittermeyer, S. Wagenpfeil, R. M. Schmid and C. Prinz, "Self-Expanding Metal Stents versus Polyethylene Stents for Palliative Treatment in Patients with Advanced Pancreatic Cancer,” Pancreas, Vol. 38, No. 1, 2009 pp. e7-e12. http://dx.doi.org/10.1097/MPA.0b013e3181870ab8

[33] T. C. Tham, D. R. Lichtenstein, J. Vandervoort, R. C. Wong, A. Slivka, P. A. Banks, H. B. Yim and D. L. CarrLocke, "Pancreatic Duct Stents for Obstructive Type Pain in Pancreatic Malignancy," The American Journal of Gastroenterology, Vol. 95, No. 4, 2000, pp. 956-960.

[34] Y. Liu, Z. Lu, D. W. Zou, Z. D. Jin, F. Liu, S. D. Li, X. B. Zhan, W. J. Zhang, R. P. Wu, Y. Z. Yao, L. Yang and Z. $\mathrm{Li}$, "Intraluminal Implantation of Radioactive Stents for Treatment of Primary Carcinomas of the PeripancreaticHead Region: A Pilot Study,” Gastrointestinal Endoscopy, Vol. 69, No. 6, 2009, pp. 1067-1073. http://dx.doi.org/10.1016/j.gie.2008.08.033

[35] K. T. Suk, J. W. Kim, H. S. Kim, S. K. Baik, S. J. Oh, S. J. Lee, H. G. Kim, D. H. Lee, Y. H. Won and D. K. Lee, "Human Application of a Metallic Stent Covered with a Paclitaxel-Incorporated Membrane for Malignant Biliary Obstruction: Multicenter Pilot Study,” Gastrointestinal Endoscopy, Vol. 66, No. 4, 2007, pp. 798-803. http://dx.doi.org/10.1016/j.gie.2007.05.037

[36] G. Morris-Stiff, A. Hassn and W. T. Young, "Self-Expanding Metal Stents for Duodenal Obstruction in Advanced Pancreatic Adenocarcinoma," HPB (Oxford), Vol. 10, No. 2, 2008, pp. 134-137. http://dx.doi.org/10.1080/13651820801938891

[37] W. A. Ross. S. M. Wasan, D. B. Evans, R. A. Wolff, L. V. Trapani, G. A. Staerkel, T. Prindiville and J. H. Lee, "Combined EUS with FNA and ERCP for the Evaluation of Patients with Obstructive Jaundice from Presumed Pancreatic Malignancy," Gastrointestinal Endoscopy, Vol. 68, No. 3, 2008, pp. 461-466. http://dx.doi.org/10.1016/j.gie.2007.11.033

[38] T. E. Yusuf, S. Ho, D. A. Pavey, H. Michael and F. G. Gress, "Retrospective Analysis of the Utility of Endoscopic Ultrasound-Guided Fine-Needle Aspiration (EUSFNA) in Pancreatic Masses, Using a 22-Gauge or 25Gauge Needle System: A Multicenter Experience,” Endoscopy, Vol. 41, No. 5, 2009, pp. 445-448. http://dx.doi.org/10.1055/s-0029-1214643

[39] B. G. Turner, S. Cizginer, D. Agarwal, J. Yang, M. B. Pitman and W. R. Brugge, "Diagnosis of Pancreatic Neoplasia with EUS and FNA: A Report of Accuracy,” Gastrointestinal Endoscopy, Vol. 71, No. 1, 2010, pp. 91-98. http://dx.doi.org/10.1016/j.gie.2009.06.017

[40] T. M. O’Toole and N. Schmulewitz, "Complication Rates of EUS-Guided Celiac Plexus Blockade and Neurolysis: Results of a Large Case Series,” Endoscopy, Vol. 41 No. 7, 2009, pp. 593-597. http://dx.doi.org/10.1055/s-0029-1214868

[41] Z. Jin, Y. Du, Z. Li, Y. Jiang, J. Chen and Y. Liu, “Endoscopic Ultrasonography-Guided Interstitial Implantation of Iodine 125-Seeds Combined with Chemotherapy in the Treatment of Unresectable Pancreatic Carcinoma: A Prospective Pilot Study,” Endoscopy, Vol. 40, No. 4, 2008, pp. 314-320. http://dx.doi.org/10.1055/s-2007-995476

[42] J. R. Hecht, R. Bedford, J. L. Abbruzzese, S. Lahoti, T. R. Reid, R. M. Soetikno, D. H. Kirn and S. M. Freeman, “A Phase I/II Trial of Intratumoral Endoscopic Ultrasound Injection of ONYX-015 with Intravenous Gemcitabine in Unresectable Pancreatic Carcinoma," Clinical Cancer Research, Vol. 9, No. 2, 2003, pp. 555-561.

[43] S. Mulvihill, R. Warren, A. Venook, A. Adler, B. Randlev, C. Heise and D. Kirn, "Safety and Feasibility of Injection with an E1B-55kDa Gene-Deleted, Replicationselective Adenovirus (ONYX-015) into Primary Carcinomas of the Pancreas: A Phase I Trial," Gene Therapy, Vol. 8. No. 4, 2001, pp. 308-315. http://dx.doi.org/10.1038/sj.gt.3301398

\author{
Abbreviations \\ EUS: Endosonography; \\ FNA: Fine Needle Aspiration; \\ AIP: Autoimmune pancreatitis.
}

